\title{
Carnets
}

Revue électronique d'études françaises de l'APEF

Première Série - 2 Numéro Spécial 10-11 | 2011

D'un Nobel l'autre

\section{Gestes et visages. Nicolas Bouvier et le regard de l'autre}

\section{Lénia Marques}

\section{OpenEdition}

1 Journals

\section{Édition électronique}

URL : http://journals.openedition.org/carnets/5776

DOI : 10.4000/carnets.5776

ISSN : 1646-7698

Éditeur

APEF

\section{Édition imprimée}

Date de publication : 1 janvier 2011

Pagination : 243-254

Référence électronique

Lénia Marques, « Gestes et visages. Nicolas Bouvier et le regard de l'autre », Carnets [En ligne],

Première Série - 2 Numéro Spécial 10-11 | 2011, mis en ligne le 16 juin 2018, consulté le 30 avril 2019. URL : http://journals.openedition.org/carnets/5776 ; DOI : 10.4000/carnets.5776

\section{(c) $(1) \Theta$}

Carnets est mis à disposition selon les termes de la licence Creative Commons - Atribution - Pas d'utilisation commerciale 4.0 International. 


\title{
GESTES ET VISAGES \\ Nicolas Bouvier et le regard de l'autre
}

LÉNIA MARQUES

CEMRI - Universidade Aberta lenia.marques@ua.pt

\begin{abstract}
Résumé
Depuis très tôt dans sa vie, Nicolas Bouvier (Genève, 1929-1998) s'est consacré au voyage et à l'écriture. Cette analyse porte sur le regard de l'autre dans l'œuvre écrite et photographique du voyageur. Écriture et photographie sont deux modes d'expression qui se complètent et qui jouent à merveille la fonction de véhicule des soucis de l'écrivain suisse envers l'Humanité, tout en étant de véritables témoins d'une philosophie de vie qui embrasse et se nourrit de la rencontre entre cultures. Le geste et le visage jouent un rôle fondamental dans l'éternel cheminement vers la découverte de la richesse de l'être humain.
\end{abstract}

\section{Abstract}

From early on, Nicolas Bouvier (Geneva, 1929-1998) dedicated himself to travel and writing. This paper proposes to investigate Bouvier's look on alterity through his writings and photographs, two complementary forms of expression used to put out his concerns towards humankind. Furthermore, writing and photography bear witness to a philosophy of life which embraces and nurtures itself from the encounter of cultures. The gesture and the face play a primal role in the continuous process of unearthing human being's richness.

Mots-clés: Nicolas Bouvier, Voyage, Photographie, Écriture, Visages, Rencontre, Altérité

Keywords: Nicolas Bouvier, Travel, Photography, Writing, Faces, Encounter, Alterity 
Cependant n'oublie pas que c'est au monde, au monde entier que tu es né, que tu dois naître, à sa vastitude.

À l'infini ton immense, dure, indifférente parenté.

Henri Michaux

... et ce bénéfice est réel, parce que nous avons droit à ces élargissements, et, une fois ces frontières franchies, nous ne redeviendrons jamais plus tout à fait les misérables pédants que nous étions.

Emerson

Un des plus grands partages de Nicolas Bouvier (Genève, 1929-1998) ${ }^{1}$ consiste dans sa philosophie du voyage qui peut se résumer en quelques mots:

On ne voyage pas pour se garnir d'exotisme et d'anecdotes comme un sapin de Noël, mais pour que la route vous plume, vous rince, vous essore, vous rende comme ces serviettes élimées par les lessives qu'on vous tend avec un éclat de savon dans les bordels. (Bouvier, 1996: 53-54)

La route, le voyage réduisent et éliminent les traits de superficialité. Tout ce dont on n'a pas besoin est progressivement éliminé, que ce soit d'une façon plus ou moins violente et chaotique, que ce soit dans un silence et une attente à peine perceptibles. L'être humain atteindrait ainsi par le biais du processus du voyage un état plus pur, plus naturel et plus en harmonie avec ce qui l'entoure.

C'est dans ce processus que nous cherchons à comprendre quel est cet autre qui se place au cœur de l'œuvre de Bouvier, œuvre qui ne pourrait exister sans cette altérité toujours présente, ne fût-ce que de manière voilée. Ainsi, cette étude se propose d'analyser le regard de l'autre dans l'œuvre scripturale et visuelle de Nicolas Bouvier. Écriture et photographie sont en effet deux modes d'expression qui accompagnent le voyage, et même le dépassent dans une certaine mesure.

Le rapport à l'autre est souvent temporaire, encore que riche de signifiés et d'émotions. Les gestes et les visages jouent dans ce cadre un rôle prépondérant. Cette analyse cherchera ainsi à dessiner les contours d'un voyage empreint d'un large éventail de gestes et de visages, pont entre gens et cultures, qui gagne de l'expression par le mot et par l'image; par la plume et par le déclencheur.

\footnotetext{
${ }^{1}$ Cet article s'encadre dans un projet de post-doctorat subventionné par la Fundação para a Ciência e a Tecnologia, dans le cadre du Programa Operacional Ciência, Tecnologia e Inovação (POCTI), Quadro de Apoio III (POCTI-SFA-18-500).
} 


\section{Nicolas Bouvier révélé}

Nicolas Bouvier, voyageur, écrivain, journaliste, photographe, iconographe, commença à voyager et à écrire très tôt dans sa vie. L'acte d'écriture, d'abord sous la forme épistolaire, a accompagné le voyage depuis son début. ${ }^{2}$ De même pour la littérature et les réflexions qui en découlent. Blaise Cendrars, Bruce Chatwin, Charles-Albert Cingria, Henri Michaux, Henri Miller, Kenneth White, Montaigne, Vladimir Holan, parmi tant d'autres, sont des références parsemées de manière récurrente dans l'œuvre comme dans la vie de Bouvier.

Le premier grand voyage en état nomade se fait en Asie. C'est en 1953, en Fiat Topolino, que le départ de Genève a lieu. Le but est d'abord de rencontrer, en Yougoslavie, son ami peintre, Thierry Vernet, pour ensuite continuer le voyage. Vernet décrit en ces termes la rencontre qui marque le début du chemin ensemble:

Le voilà, pâle et les traits tirés. La route était longue et il a fait très chaud. On se jette dans les bras l'un de l'autre, très émus. On ne dit rien. On se reprend. II va se laver les mains. Ouf! Je dis ouf! C'est tout un monde qui arrive avec lui [...]. (Vernet, 2006: 86)

Après quatre ans de route, Bouvier retourne seul en Suisse. En effet, ce grand voyage peut se diviser en quatre étapes, bien marquées littérairement: la première, avec Vernet jusqu'au Khyber Pass, correspond à son premier récit L'Usage du monde; la deuxième est la descente de l'Inde, tout seul en voiture, et dont il ne reste que quelques textes épars et des émissions radiophoniques; ${ }^{3}$ la troisième consiste en son séjour à Ceylan, prolongé sur plusieurs mois, et qui, une vingtaine d'années plus tard, a donné origine au récit Le Poisson-Scorpion; la dernière correspond à son premier séjour au Japon, qui, avec d'autres, ${ }^{4}$ aura comme résultat Japon / Chronique japonaise. ${ }^{5}$

Entre le déplacement physique et l'acte d'écriture, il y a souvent un décalage temporel significatif. L'acte d'écriture exige du temps, il est douloureux, voire maladif. Tel l'a été le processus de fabrication du Poisson-Scorpion:

\footnotetext{
${ }^{2}$ La correspondance échangée avec Thierry Vernet en est un des meilleurs exemples (Bouvier et Vernet, 2010).

3 À ce propos, cf. Bouvier, 2004b: 433-494; Laut, 2008: 101-108.

${ }^{4}$ Bouvier y retournera en effet plusieurs fois: "je savais que j'y retournerais. J'y avais laissé mon cœur" (Bouvier, 2004d: 1331). Sur le Japon de Nicolas Bouvier, cf. Shoda-Fujizane (2007: 143-154).

${ }_{5}$ Japon est le titre d'une publication commandée qui réunit photographie et texte (Bouvier, 1967). Quelques années plus tard, le voyageur-écrivain la reprend avec quelques changements et lui donne le titre de Chronique japonaise, livre qui a d'ailleurs connu des versions quelque peu différentes (Bouvier, 1997).
} 
J'ai écrit ce livre quasiment en transe, sur des flots de whisky et de musique. [...] J'étais obligé de l'écrire pour me débarrasser de ce mal. [...] C'était vraiment ce que j'appelle 'l'écriture-exorcisme'.“ (Bouvier, 2004d: 1330)

Si Nicolas Bouvier est acclamé comme un jalon de la littérature de voyages, ses écrits sont célébrés par leur beauté. Pourtant, ses premiers pas ont été bien difficiles. Vers le début des années soixante, Bouvier se voit refuser la publication de L'Usage du monde. C'est donc à compte d'auteur qu'en 1963 paraît le récit avec des dessins de Thierry Vernet. Comme l'a affirmé Bouvier, Vernet et lui avaient deux approches, deux manières de percevoir la même réalité. Les dessins dans L'Usage du monde ne se limitent pas à une illustration. Leur présence établit "une sorte de dialogue entre l'image et le texte" (Bouvier, 1963). Ainsi, déjà dans l'approche de ce travail conjoint entre mot et image, la posture de l'auteur commence à se révéler. Plus tard, et pour son propre travail de photographe et même d'iconographe, sa philosophie des rapports entre texte et image parvient à s'afficher d'une manière plus accentuée.

Si l'on revient à L'Usage du monde, sa réception en 1963 a plutôt été un échec. Ce ne sera donc qu'en 1981, avec la parution du Poisson-Scorpion, que Bouvier commencera d'être connu dans la littérature de voyage. En 1985, la réédition de L'Usage du monde marque définitivement le succès de Bouvier, voyageur-écrivain. D'ailleurs, en 1986, l'écrivain genevois reçoit le Prix des Belles-Lettres et l'année suivante il se voit reconnu par le Prix de la Ville de Genève. D'autres titres et d'autres prix ont suivi.

Jusqu'à sa mort en 1998, Nicolas Bouvier continua de publier et d'entamer plusieurs projets. Non seulement il a longtemps eu comme métier être "chercheur d'images" (Bouvier, 2001a: 49), mais, vers les dernières années de sa vie, le dialogue entre texte et image semble se faire d'une manière plus puissante, dans le sens où le voyageur cherche progressivement à faire des ensembles signifiants entre ses textes et ses images. Certains de ces projets n'ont aboutit qu'après sa mort, donnant ainsi lieu à des publications posthumes auxquelles Bouvier a en effet activement participé. ${ }^{6}$

L'Usage du monde et Le Poisson-Scorpion sont les titres les plus vastement connus, mais l'écrivain-voyageur nous a aussi laissé de belles pièces d'écriture comme Japon / Chronique japonaise, Journal d'Aran, L'Échappée belle ou le court et dense livre de poèmes Le Dehors et le dedans. L'ensemble de son œuvre, mais surtout sa philosophie de vie et sa philosophie du voyage, ont inspiré d'autres à produire différents ouvrages (souvent après un départ) qui vont du film documentaire à I'hommage par l'écrit et/ou par la photographie. ${ }^{7}$

\footnotetext{
${ }^{6}$ Cf. eg. Bouvier (1998).

7 Cf. Bouvier et Plattner (1993); Kühn (2005); Rechsteiner (2005); Calmettes et Bauer (2008); Lecloux (2008); Métroz (2008).
} 
Donc, derrière l'acte de prendre la plume ou d'appuyer sur son déclencheur, Bouvier s'est construit une philosophie de vie, dans laquelle ses principes sur le voyage sont compris.

\section{Notes pour une philosophie du voyage}

Une des questions que l'on pourrait se poser concerne exactement l'apport de Bouvier à la littérature de voyage. Finalement, il n'a pas été le seul à mener à bout des voyages en Orient, bien au contraire. Or, une des particularités les plus marquées de Bouvier est sa conception du voyage, reflet de ses pratiques. Avant de se considérer comme photographe ou écrivain, Nicolas Bouvier se voit avant tout comme un voyageur. Voilà donc le point de départ constant dans son œuvre. Le voyage, n'importe lequel, que ce soit à l'autre bout du monde ou juste quelques mètres plus loin, vit en lui et marque sans aucun doute le parcours de sa vie.

Le voyage est ainsi vécu intensément, mais il est le contraire d'une aventure exotique. En effet, le "regard du voyageur de Bouvier ne se situe pas sur le même plan que celui du regard distrait et superficiel du touriste en quête d'exotisme" (Laurel, 2006: 200). L'auteur détrompe d'ailleurs son lecteur lorsqu'il écrit, tout au début de L'Usage du monde, dans un extrait vastement connu: "Un voyage se passe de motifs. II ne tarde pas à prouver qu'il se suffit à lui-même. On croit qu'on va faire un voyage, mais bientôt c'est le voyage qui vous fait, ou vous défait” (Bouvier, 2001b: 12).

C'est ainsi que dès son premier grand récit, le voyageur genevois souligne l'action inéluctable du voyage sur le sujet, une action qui se révèle à double tranchant: il construit comme il détruit. Les motifs sont ainsi réduits à l'insignifiance, puisque le binôme construction/destruction se passe moins au niveau extérieur (objets observés) qu'au niveau intérieur (construction du sujet). Plutôt que par un acte de révolte ou poussé par le mirage d'un répertoire exhaustif de motifs, le voyage se fait par besoin d'autre chose: "J'ai pris le large parce que j'en avais besoin, mais c'était une fuite positive, une course vers des choses dont j'avais besoin, que j'ai cherchées et trouvées" (Bouvier, 2004d: 1341).

Le voyage est ainsi le moyen d'ouvrir de nouveaux horizons, de chercher son épanouissement en tant qu'être humain; de connaître le monde pour mieux pouvoir le recevoir: "Le voyage devient ainsi l'espace de la découverte de l'essentiel, de la quête du sens de ce qui remplit nos journées [...]. Quête qui exige la fusion du narrateur-voyageur dans le monde" (Laurel, 2006: 203). Le voyage n'est aucunement un épisode dans la vie du sujet, il "est avant tout un état d'esprit avant d'être une pratique spatiale" (Ridon, 2007: 10); il constitue une véritable façon d'être dans le monde: "Pour Vernet et pour moi, le voyage était une façon de vivre, un mode de vie plus intense et lyrique que la vie sédentaire [mais] qui n'est pas le contraire de la vie sédentaire" (Bouvier, 1963). C'est pour cette raison que 
s'affirmant toujours comme le voyageur qu'il était, Nicolas Bouvier a gardé des liens en Suisse, son "port d'attache". 8

Au fond, le voyage fait partie d'une quête incessante dont le sujet ne peut qu'espérer atteindre des moments de pleine extase et d'harmonie parfaite avec le monde qui l'entoure: "On voyage pour faire apparaître le monde et connaître avec lui, comme avec une femme, de trop brefs instants d'unité indicible et de totale réconciliation" (Bouvier, 1996: 50). Unité qui ne peut être dite - limite du pouvoir des mots - et réconciliation absolue avec le monde: fusion momentanée entre le dehors et le dedans, rare et pour cela même précieuse, mémorable, indicible, secrète. Le silence de Bouvier sur son séjour en Inde peut trouver dans l'indicibilité sa raison d'être...

C'est à souligner donc le caractère transformateur du voyage, qui fait apparaître le monde et qui permet non seulement de le connaître, mais d'entrer dans une intimité profonde avec lui. Voilà grande ouverte la porte qui sépare intérieur et extérieur, dedans et dehors.

\section{Le regard de l'autre}

Le voyage permet ainsi de construire des ponts, d'établir des liens entre le sujet et autrui, entre le sujet et le monde environnant. Que ce soit du point de vue de l'écriture, que ce soit par rapport à la photographie, le regard porté sera toujours vers l'extérieur et nécessairement subjectif. C'est dans ce sens que Jean-Xavier Ridon écrit que "Bouvier ne cachera jamais le lieu à partir duquel il prend la parole, à savoir, et pour caricaturer un peu, celle d'un jeune Blanc en vacances. II ne prétendra jamais non plus parler pour les autres" (Ridon, 2007: 10). Donc, le voyageur genevois est bien conscient de sa position et de sa place dans des endroits différents.

II est l'étranger, celui qui n'est pas dans son lieu d'origine, qui est en dehors de cet univers spécifique. En effet, il note dans Chronique japonaise: "Et puis, c'est un concept ambigu, l'étranger, et dont on peut aisément inverser le signe" (Bouvier, 2004a: 641). II est en même temps cet être déterritorialisé, détaché de son environnement familier. Cet exercice de détachement, d'épuration et de fécondation de liens renouvelés au monde se manifeste dans le regard de l'autre. Au cœur de cette formule - regard de l'autre - se trouve l'ambivalence fertile de la préposition "de". L'œuvre de Bouvier témoigne profusément de la même ambivalence en ce qui concerne le regard. En effet, le "regard de l'autre" comprend simultanément l'autre que Nicolas Bouvier regarde et lui-même, étranger qui est regardé, c'est-à-dire qui est objet du regard de l'autre.

\footnotetext{
8 "J'ai toujours eu de longues périodes de sédentarisme dans cette maison: tout bateau a besoin d'une cale sèche et d'un port d'attache dont le nom figure sur l'étrave" (Bouvier, 2004d: 1380).
} 
Ainsi, sa manière de voir et de raconter le monde, son Histoire et celle de ses gens, passe dans une large mesure par le regard et la perception de l'altérité - deux éléments qui, mis ensemble, résultent tout au moins en deux mouvements différents: le premier, le regard qui va de celui qui s'exprime (l'écrivain ou le photographe) à celui qui est observé; le second, la présence nette du regard de cet autre porté vers le sujet qui s'exprime. Ainsi, chez Bouvier, l'observateur occupe souvent aussi la place de l'objet observé. En outre, un autre phénomène a souvent lieu: la co-présence de ces deux mouvements. En effet, plus qu'opposés, ils sont montrés et vécus comme complémentaires, encore qu'ils se situent à des niveaux tout à fait distincts.

Toutefois, et vu que l'écrivain assume explicitement son point de vue (bien qu'il tente d'éviter une présence égocentrique et fermée), il est bien évident que le regard que l'autre porte sur Bouvier est paradoxalement exprimé par Bouvier lui-même. En écriture, ce processus laisse plus de traces qu'en photographie, même si le regard perçu dans la pellicule est soumis à des choix subjectifs toujours opérés par Bouvier. Ainsi en est-il que, en dernière instance, l'expression du regard de cet autre est toujours le résultat d'un regard sur l'autre: Bouvier regarde celui qui le regarde regarder. ${ }^{9}$ L'acte est un jeu: d'abord, la conversation; puis un accord; et finalement l'entrée dans le jeu avec gaieté (cf. Bouvier, 1984). Nous nous trouvons donc face à un mouvement circulaire et riche, mais toujours subjectif. C'est d'ailleurs ce qui rend l'expérience encore plus captivante. Dans ce sens, Bouvier va au-delà de la simple description et de l'anecdote basée sur une observation qui se veut dénouée, épurée, sans contraintes, objective. Or Bouvier va plus loin dans le sens où, malgré les différences avouées et parfaitement conscientes entre lui et cet autre, il cherche sans cesse à repérer le point où la communion est (serait) possible.

En même temps, l'expérience de l'altérité et du monde environnant en est une du passage: le temps qui passe, le monde qui change, la vie qui continue. L'être humain, et dans le cas spécifique de Bouvier, le voyageur, est confronté en permanence à un sentiment de vide et de plein: ${ }^{10}$

Comme une eau, le monde vous traverse, et, pour un temps, vous prête ses couleurs. Puis, se retire, et vous replace devant ce vide qu'on porte en soi, devant cette espèce d'insuffisance centrale de l'âme qu'il faut bien apprendre à côtoyer, à combattre, et qui, paradoxalement, est peut-être notre moteur le plus sûr. (Bouvier, 2001b: 418)

\footnotetext{
${ }^{9}$ Les portraits que l'on peut contempler dans Le Japon de Nicolas Bouvier, par exemple, en sont de beaux exemples (Bouvier, 2002).

${ }^{10}$ À ce propos, référons les notes de Bouvier sur le Japon qui ont été publiées sous le titre Le Vide et le plein (Bouvier, 2009).
} 
Au cœur de ce mouvement de traversée, l'écriture est un moyen de raconter, certes trop lent et parfois ingrat. La photographie surgit alors comme une solution différente, moins difficile, moins lente et avec des caractéristiques qui permettent d'exprimer ce que les mots parfois n'ont pas le pouvoir de dire. Écriture et photographie correspondent à des états d'esprit, des besoins et des urgences différents. L'écriture est un acte lent, voire rituel, obligé lors d'une pression trop forte que Bouvier n'est plus en mesure de contrôler; pour la photographie, il faut tout un entraînement, perceptif et technique aussi: "Il y a une interaction très intéressante entre la photo et l'écriture, mais on ne peut pas les pratiquer dans le même temps. [...] [Pendant son séjour au Japon] II y avait des jours pour la photo et des jours pour le texte. Je le sentais en me levant" (Bouvier, 2004d: 1314).

Selon Laurence Guyon, "[i]l semble que face aux lacunes et aux imperfections de la pratique littéraire, l'alternative soit photographique. Un des modes d'accès privilégié à ce visage du monde qui nous est dérobé, c'est peut-être précisément pour Nicolas Bouvier la photographie et plus notablement encore le portrait photographique" (Guyon, 2008: 492). Le voyageur suisse en est d'ailleurs parfaitement conscient:

Je suis portraitiste. [...] [C]e qui m'intéresse avant tout, c'est le visage des gens et encore plus, ce qui se passe entre le photographe et le modèle au moment où prend place cette opération à caractère magique.

D'abord, il faut que le photographe s'efface complètement. [...] II faut attendre de voir monter toutes les émotions qui ont été réprimées, les projets, les souvenirs. Les visages se remplissent comme une chambre vide que l'on meublerait en hâte pour un hôte inattendu. Cette émergence est extrêmement touchante. (Bouvier, 2004d: 13091310)

De nouveau apparaît le double mouvement du vide et du plein, cette fois-ci au niveau des émotions, des projets, des souvenirs - donc de la vie. La fonction du photographe, de cette figure dont le regard se cache, est de, "à force de patience et d'humilité, [...] faire apparaître le contour intérieur d'un visage" (Bouvier, 2004c: 702).

Si la photographie a ce pouvoir, dans l'écriture, les gestes, les têtes, les visages et le regard partagent de ce même esprit de révélation: "Les mâchoires plombées étincellent et les visages commencent à briller: tannés, ridés, les traits tout écornés comme ceux des bouddhas de cimetière; mais ce qu'il en reste est très doux et les regards ont quelque chose de direct et d'espiègle que je n'ai pas trouvé souvent ici [en Suisse]“ (Bouvier, 2004a: 650).

Ainsi, pour Bouvier le visage qui se révèle et la possibilité de saisir des émotions, que ce soit par la plume ou par la pellicule, acquièrent un sens qui dépasse l'expérience 
esthétique et touche à l'expérience humaine à travers la construction de ponts entre les gens et entre les cultures.

\title{
4. Des ponts solides à l'insolubilité des obstacles
}

Le visage devient une porte d'accès à l'altérité; la photographie une manière de l'exprimer: "L'image photographique implique le désir de voir plus, de voir au-delà, de voir à travers. Le portrait est, pour l'auteur, un élément totalisant, par le biais duquel il est possible d'accéder à une conversion culturelle immédiate" (Marchetti, 1994: 61). L'échange interculturel est ainsi facilité. Par ailleurs, il faut penser également que la porte d'entrée vers l'altérité se dédouble et devient le miroir d'un "je" qui se cache, qui cherche à disparaitre, mais qui est, quand même, toujours présent: "Le voyage que Nicolas Bouvier effectue vers le noyau de l'altérité le conduit dans la direction du moi en tant qu'ellipse pure" (Marchetti, 1994: 64). ${ }^{11}$

Établir des liens entre le voyageur et l'autre (normalement l'habitant du lieu) apparaît à Bouvier comme acte essentiel à un esprit ouvert. Le poème d'Hafiz que Bouvier et Vernet décident d'inscrire, en persan, sur la portière de la voiture qui les accompagnera tout au long du voyage, en est le témoin:

\author{
Même si l'abri de ta nuit est peu sûr \\ et ton but encore lointain \\ sache qu'il n'existe pas \\ de chemin sans terme \\ Ne sois pas triste \\ (cité dans Bouvier, 2001b: 251)
}

Ces mots du poète persan ont servi comme un pont interculturel effectif, un signe de paix et d'ouverture à l'autre, en même temps qu'ils affichent les principes éthiques et poétiques des voyageurs. Le poème d'Hafiz a plusieurs fois ouvert un passage vers des cultures où la communication pourrait, du moins à première vue, s'avérer difficile.

Mais tout n'est pas positif. Le voyage est un médaillon qui présente en effet, et comme Bouvier l'a largement souligné, deux côtés. D'une part, la connivence et une sorte de communion ne sont possibles que lorsqu'il y a un partage complet, du positif autant que du négatif: "On ne peut pas comprendre les gens si on ne partage pas entièrement leur vie, côté positif et côté négatif" (Bouvier, 1963). D'autre part, souvent la communion, la

\footnotetext{
${ }^{11}$ Cette ellipse est au cœur de la poétique de la disparition dans l'œuvre de Bouvier (cf. Hambursin, 1997 et Ridon, 2002).
} 
compréhension et les gestes d'empathie ne trouvent simplement pas leur place. L'intégration n'est pas toujours possible, moins encore évidente. À Ceylan, les difficultés à ce niveau sont notoires. À cet endroit, un ensemble de circonstances participe en effet à l'impossibilité affichée et permanente de tisser des liens: "Nos rapports se réduisent donc à cette tolérance réciproque, hésitante et timorée. [...] Tout de même c'est beau [...] ce lieu qui ne ressemble à rien de ce que j'ai connu, cette tanière d'où je prends les deux pouls de la ville, celui des hommes et celui des insectes" (Bouvier, 1996: 61). Ici, hommes et insectes sont observés de l'extérieur comme contribuant au même flux de vie. Outre le personnage fantomatique du Père Alvaro, la seule exception positive à la "tolérance" est la Circé, l'épicière, qui, devinant ses pensées et peut-être son cœur, lui offre le poisson-scorpion qu'il admire.

\section{Pour conclure}

L'œuvre de Bouvier, écrite comme photographique, témoigne de beaux moments de rencontre avec l'autre, moments où se donne un véritable partage d'identités et de cultures, individuelles comme collectives. Toutefois, le voyage n'est pas que succès; il nous apprend l'échec et l'humilité; l'essentiel et I'humain. Les émotions ressenties et exprimées sont toujours éléments d'un équilibre qui, lorsqu'atteint, ne peut qu'être fragile:

La littérature et la photographie, dans leur interdépendance, sont pour Nicolas Bouvier, de véritables sésames, qui ouvrent momentanément les portes de l'ineffable et permettent à l'individu qui s'absente à lui-même d'accéder à l'arrière-plan métaphysique de l'Univers, ou du moins, d'en avoir passagèrement l'intuition (Guyon, 2008: 495).

Dans ce processus, le geste et surtout le visage jouent un rôle fondamental dans l'éternel cheminement vers la découverte de l'essence de l'être humain. Tout voyage (du dehors comme du dedans) n'est finalement qu'un jeu composé de divers moments - on s'attache, on s'arrache $-{ }^{12}$ au bout duquel, pourrait-on dire avec Cingria, un autre voyageur suisse qui a inspiré Bouvier, "[i]l y aura autre chose" (Cingria, 1967-1978: 54).

\footnotetext{
12 "La dialectique de la vie nomade est faite de deux temps: s'attacher et s'arracher. On n'arrête pas de vivre ce couple de mots tout au long de la route" (Bouvier, 2004d: 1290).
} 


\section{Bibliographie}

BOUVIER, Nicolas (1963). "L'Usage du monde", Émission télévisée "À livre ouvert", TSR (Télévision Suisse Romande) du 28/11/1963, avec le journaliste Maurice Huelin, [consulté le 15/03/2010] <URL: http://archives.tsr.ch/dossier-bouvier/personnalite-bouvier2>.

BOUVIER, Nicolas (1967). Japon. s.I. Éditions Rencontre.

Bouvier, Nicolas et PlatTner, Patricia (1993). Le Hibou et la Baleine [livre et enregistrement vidéo]. Genève-Carouge: Zoé.

BOUVIER, Nicolas (1984). "L'Image et ses leçons", Émission télévisée "Visiteurs du soir", TSR (Télévision Suisse Romande) du 17/02/1984, [consulté le 15/03/2010] <URL: http://archives.tsr.ch/dossier-bouvier/personnalite-bouvier4>.

Bouvier, Nicolas (1996). Le Poisson-Scorpion [1982]. Paris: Gallimard.

BOUVIER, Nicolas (1997). Chronique japonaise [1975]. Paris: Payot.

BOUVIER, Nicolas (1998). Entre Errance et éternité. Regards sur la montagne. Genève: Zoé.

BOUVIER, Nicolas (2001a). Histoires d'une image. Genève: Zoé.

BOUVIER, Nicolas (2001b). L'Usage du monde [1963]. Paris: Payot.

BOUVIER, Nicolas (2002). Le Japon de Nicolas Bouvier. Paris: Hoëbeke.

BOUVIER, Nicolas (2004a). Chronique japonaise [1975]. In: Nicolas Bouvier. CEuvres. Paris: Gallimard, pp. 495-669.

BouvIER, Nicolas (2004b). L'Inde. In: Nicolas Bouvier. CEuvres. Paris: Gallimard, pp. 433-494.

Bouvier, Nicolas (2004c). Notes en vrac sur le visage. In: Nicolas Bouvier. CEuvres. Paris: Gallimard, pp. 701-706.

BOUVIER, Nicolas (2004d). Routes et déroutes [entretiens avec Irène Lichtenstein-Fall]. [1992] In: Nicolas Bouvier. CEuvres. Paris: Gallimard, pp. 1249-1388.

BOUVIER, Nicolas (2005). Charles-Albert Cingria en roue libre (textes réunis par Doris Jakubec). Genève: Zoé.

BOUVIER, Nicolas (2009). Le Vide et le plein. Carnets du Japon 1964-1970. [2004] Paris: Gallimard.

BOUVIER, Nicolas et VERNET, Thierry (2010). Correspondance des routes croisées. 1945-1964 (texte établi, annoté et présenté par Daniel Maggetti et Stéphane Pétermann). Carouge-Genève: Éditions Zoé.

CALMETTES, Joël et BAUER, Olivier (2008). Nicolas Bouvier, le vent des mots [1999] [DVD]. France: Arcades Vidéo (45').

CINGRIA, Charles-Albert (1967-1978). Graffiti. In: CEuvres complètes, t.VI. Lausanne: L’Âge d'Homme, pp. 52-54.

GUYON, Laurence (2008). “'C'que c'est beau la photographie!'”. In: Jean-Pierre Montier, Liliane Louvel, Danièle Méaux et Philippe Ortel (éds.) (2008). Littérature et photographie. Rennes: Presses Universitaires de Rennes, pp. 487-495.

HAMBURSIN, Olivier (1997). "Voyage et exercice de disparition: les dangers du Poisson-Scorpion de Nicolas Bouvier". In: Les Lettres romanes, n3 -4, t. LI, pp. 275-287.

KüHN, Christophe (2005). Nicolas Bouvier - 22 Hospital Street [DVD]. Suisse: Filmkollektiv (83').

LAUREL, Maria Hermínia Amado (2006). "De L'Usage du monde comme itinéraire identitaire", in Cadernos de Literatura Comparada 14/15, Textos e Mundos em deslocação, t.1, Porto: Edições Afrontamento / Instituto de Literatura Comparada Margarida Losa, pp. 185-207.

LAUT, François (2008). Nicolas Bouvier. L'CEil qui écrit. Paris: Payot.

LECLOUX, Frédéric (2008). Usure du monde: Hommage à Nicolas Bouvier. Manosque: Le Bec en l'Air.

MARCHETTI, Marilia (1994). "Voyage, mémoire et représentation dans l'œuvre de Nicolas Bouvier". In: Yves Bridel (coor.) (1994). La Suisse ouverte: Nicolas Bouvier. Cahiers francophones d'Europe Centre-Orientale, 4, A.E.F.E.C.O. 
METROZ, Gaël (2008). Nomad's land: Sur les traces de Nicolas Bouvier [DVD]. Suisse: Tipi'mages Productions (90').

RECHSTEINER, Eric (2005). Indigo street: sur les routes de Nicolas Bouvier (préface de Gilles Lapouge et textes de Nicolas Bouvier). s.I. Éditions de la Boussole.

RIDON, Jean-Xavier (2002). "Pour une poétique du voyage comme disparition". In: N. Laporte, C. Albert et J.-Y. Pouilloux (2002). Autour de Nicolas Bouvier. Résonances. Carouge-Genève: Zoé, pp. $120-135$.

RIDON, Jean-Xavier (2007). Le Poisson-Scorpion. Nicolas Bouvier, Carouge-Genève: Zoé.

SHODA-FUJIZANE, Yasuko (2007), "Invitation à la 'vie immédiate': Chronique japonaise de Nicolas Bouvier". In Actes du Forum APEF 2006. Espaces de la Francophonie en débat [online]. APEF, pp. 143-154 [consulté le 15/03/2010] <URL: http://www.apef.org.pt/actas2006/YF122006.pdf>.

VERNET, Thierry (2006). Peindre, écrire. Chemin faisant. Lausanne: L'Âge d'Homme. 